Zeitschrift für Physik, Bd. 147, S. 628-635 (1957)

Aus dem Institut für Theoretische Physik der Universität Heidelberg

\title{
Zur Theorie der Konversionskoeffizienten
}

[Berichtigung und Ergänzung zu Z. Physik 146, 187-204 (1956)]

\author{
Von \\ GUSTAV KRAMER \\ (Eingegangen am 19. Dezember 1956)
}

\begin{abstract}
Eine in einer früheren Note angegebene Formel für die Korrektur von elektrischen Konversionskoeffizienten wird berichtigt. Ferner wird der Zusammenhang der dort abgeleiteten Gleichungen für die Wechselwirkung von Elektronen und $\mathrm{Nu}$ kleonen mit dem von H.R. HuLME entwickelten retardierten Wechselwirkungsoperator hergestellt.
\end{abstract}

\section{§1. Berichtigung}

Herr Professor M.E. Rose (Oak Ridge, Tennessee) und Herr Professor TH.A. Green (Middletown, Connecticut) haben mich darauf aufmerksam gemacht, daß der Ausdruck für $\delta(L$, elektr) in Gl. (51) auf S. 202 der genannten Arbeit ${ }^{1}$ nicht korrekt ist.

$\delta(L$, elektr) berechnet man, indem man von G1. (38) ausgeht. In Gl. (51) müssen daher die Potentiale aus Gl. (38) stehen. $\delta(L$, elektr) lautet also:

$$
\begin{aligned}
& \delta(L, \text { elektr })= \\
& \frac{\sum_{n=1}^{A}\left\{\int_{0}^{R}\left[\psi_{f}^{K^{*}} \vec{\alpha}_{n} \vec{B}_{L M}^{\mathrm{e} *^{*}}\left(W v_{n}\right) \psi_{i}^{K} \int_{0}^{\gamma_{n}} \psi_{f}^{E^{*}}\left\{\vec{\alpha}_{E} W \mathfrak{r}_{E}-i \frac{d}{d \gamma_{E}} \gamma_{E}\right\} \frac{\Phi_{L M}\left(W v_{E}\right)}{\sqrt{L(L+1)}} \psi_{i}^{E} d \tau_{E}\right\} d \tau_{K}\right\}}{\sum_{n=1}^{A} \int_{0}^{R} \psi_{f}^{K^{*}} \vec{\alpha}_{n} \vec{A}_{L M}^{\mathrm{e}{ }^{*}}\left(W \gamma_{n}\right) \psi_{i}^{K} d \tau_{K}} \\
& -\frac{\sum_{n=1}^{A}\left\{\int_{0}^{R}\left[\psi_{f}^{K^{*}} \vec{\alpha}_{n} \vec{A}_{L M}^{\mathrm{el}^{*}}\left(W r_{n}\right) \psi_{i}^{K} \int_{0}^{\gamma_{n}} \psi_{f}^{E^{*}}\left\{\vec{\alpha}_{E} W \mathfrak{r}_{E}-i \frac{d}{d \gamma_{E}} \gamma_{E}\right\} \frac{\psi_{L M}\left(W \gamma_{E}\right)}{\sqrt{L(L+1)}} \psi_{i}^{E} d \tau_{E}\right] d \tau_{K}\right\}}{\sum_{n=1}^{A} \int_{0}^{R} \psi_{f}^{K^{*}} \vec{\alpha}_{n} \vec{A}_{L M}^{\mathrm{e}^{*}}\left(W \gamma_{n}\right) \psi_{i}^{K} d \tau_{K}},
\end{aligned}
$$

Den Herren Professor Rose und Professor Green danke ich vielmals für ihre Mitteilung.

1 Kramer, G.: Z. Physik 146, 187 (1956), im folgenden als I zitiert. 
Außerdem sind einige Druckfehler in I zu berichtigen. In Gl. (23) gibt $\mu$ den Index der BeSSEL- bzw. HANKEL-Funktionen an. In Gl. (28) ist der Faktor $k / W$ zu streichen und in Gl. (37) muß es an Stelle von

$$
\left\{\vec{\alpha}_{E} W^{2} \mathfrak{r}_{E}-i W \frac{d}{d r_{E}} r_{E}\right\} \text { heißen: } \frac{1}{W}\left\{\vec{\alpha}_{E} k^{2} \mathfrak{r}_{E}-i W \frac{d}{d r_{E}} r_{E}\right\} \text {. }
$$

\section{§2. Der Wechselwirkungsoperator zwischen Elektronen und Nukleonen}

Anstatt die Störungsrechnung von vornherein durch Entwicklung der Potentiale des Strahlungsfeldes nach Multipolen durchzuführen, lassen sich die in I angegebenen Formeln für die Konversionskoeffizienten auch unmittelbar aus einem bereits von HuLME ${ }^{2}$ angegebenen Ausdruck für das Matrixelement $U_{f i}(W)$, das die Übergangswahrscheinlichkeit für Konversion bestimmt, gewinnen. Die Hulmesche Forme lautet*:

$U_{f i}(W)=\sum_{n=1}^{A} e_{E} e_{n} \int \psi_{f}^{K^{*}} \psi_{f}^{E^{*}}\left(1-\vec{\alpha}_{n} \vec{\alpha}_{E}\right) \frac{e^{i W\left|\mathfrak{r}_{n}-\mathfrak{r}_{E}\right|}}{\left|\mathfrak{r}_{n}-\mathfrak{r}_{E}\right|} \psi_{i}^{K} \psi_{i}^{E} d \tau_{E} d \tau_{K}$.

$U_{f i}(W)$ hat dieselbe Gestalt wie das Übergangsmatrixelement für die Streuung zweier freier Elektronen** in der halbklassischen Theorie von $\mathrm{M} \varnothing \mathrm{LLER}^{3}$ mit retardierten Potentialen.

Wegen der Einfachheit und allgemeinen Anwendbarkeit des Hulmeschen Ausdruckes sei eine einfache Herleitung gegeben, bei der die Entwicklung des elektromagnetischen Feldes nach Multipolen noch nicht erforderlich ist. Im Anschluß an Hulme benutzen wir wie in I die Coulomb-Eichung, d.h. wir führen die Coulombsche Wechselwirkung explizit in den Hamilton-Operator ein. Für die Potentiale $\mathfrak{A}(\mathfrak{r})$ des Strahlungsfeldes gilt dann die spezielle LoRENTz-Bedingung $\operatorname{div} \mathfrak{A}=0$. Der Wechselwirkungsoperator $H$ hat dann die Gestalt [s. (4)]:

$$
H=e_{E} \vec{\alpha}_{E} \mathfrak{U}\left(\mathfrak{r}_{E}\right)+\sum_{n=1}^{A}\left\{e_{n} \vec{\alpha}_{n} \mathfrak{A}\left(\mathfrak{r}_{n}\right)+\frac{e_{n} e_{E}}{\left|\mathfrak{r}_{n}-\mathfrak{r}_{E}\right|}\right\} .
$$

Der Einfachheit halber wollen wir von den Beiträgen der anomalen magnetischen Momente zu $H$ absehen.

* Die Definition der gebrauchten Symbole findet man in I. - Wegen des engen Zusammenhanges mit I setzen wir die Numerierung der Formeln im AnschluB an I fort.

** Im Falle von zwei Elektronen muß noch der Anfangs- oder der Endzustand antisymetrisiert werden.

2 Hulme, H.R.: Proc. Roy. Soc. Lond., Ser. A 154, 487 (1936).

3 Mrslez, C.: Ann. d. Phys. 14, 531 (1932). 
Das elektromagnetische Potential $\mathfrak{A}(\mathfrak{r})$ entwickeln wir jetzt nach ebenen Wellen:

$$
\mathfrak{A}(\mathfrak{r})=\frac{1}{(2 \pi)^{\frac{3}{2}}} \sum_{i=1}^{2} \int d^{\mathfrak{3}} \mathfrak{\mathfrak { l }}\left\{q_{i}(\mathfrak{\mathfrak { t }}) \mathfrak{e}_{i} e^{i \mathfrak{k} \mathfrak{r}}+q_{i}^{*}(\mathfrak{l}) \mathfrak{e}_{i} e^{-i \mathfrak{k} \mathfrak{r}}\right\}
$$

Die Vektoren $\mathfrak{e}_{i}$ geben die beiden möglichen Polarisationstichtungen für die transversalen Potentiale an. $\mathfrak{e}_{1}, \mathfrak{e}_{\mathbf{2}}$ und $\mathfrak{l} / k$ bilden ein Dreibein. $U_{f i}(W)$ ergibt sich dann aus (15) unter Berücksichtigung von (13) für die Vernichtungs- und Erzeugungsoperatoren $q_{i}(\mathfrak{t})$ und $q_{i}^{*}(\mathfrak{f})$ und den in I, Tabelle 1, S. 192, angegebenen Möglichkeiten für die $Z$ wischenzustände. Man erhält:

$$
\begin{aligned}
& U_{f i}(W)=\sum_{n=1}^{A} e_{n} e_{E}\left\{\int \psi_{f}^{K^{*}} \psi_{f}^{E^{*}} \frac{1}{\left|\mathfrak{r}_{n}-\mathfrak{r}_{E}\right|} \psi_{i}^{E} \psi_{i}^{K} d \tau_{E} d \tau_{K}+\right. \\
& +\lim _{\sigma \rightarrow 0} \frac{1}{4 \pi^{2}} \sum_{i=1}^{2}\left[\int d^{3} \mathfrak{i} \frac{1}{k(W-k+i \sigma)} \times\right. \\
& \times \int \psi_{f}^{E^{*}} \vec{\alpha}_{E} \mathrm{e}_{i} e^{i \mathfrak{t} \mathfrak{r}_{E}} \psi_{i}^{E} d \tau_{E} \int \psi_{f}^{K^{*}} \vec{\alpha}_{u} \mathrm{e}_{i} e^{-i \mp \mathfrak{r}_{n}} \psi_{i}^{K} d \tau_{K}- \\
& \left.\left.--\int d^{3 \mathfrak{k}} \frac{1}{k(W+k+i \sigma)} \int \psi_{\dot{f}}^{E^{*} \vec{\alpha}_{E}} \mathfrak{e}_{i} e^{-i \mathfrak{k} \mathfrak{r}_{E}} \psi_{\dot{i}}^{E} d \tau_{E} \int \psi_{\dot{f}}^{K^{*}} \vec{\alpha}_{n} \mathfrak{e}_{i} e^{i \mathfrak{f} \mathfrak{r}_{n}} \psi_{i}^{K} d \tau_{K}\right]\right\} .
\end{aligned}
$$

Die Summation über die Polarisationsrichtungen können wir sofort auf Grund der Formel:

$$
\sum_{i=1}^{2}\left(\vec{\alpha}_{E} \mathrm{e}_{i}\right)\left(\vec{\alpha}_{n} e_{i}\right)=\left(\vec{\alpha}_{E} \vec{\alpha}_{n}\right)-\frac{1}{k^{2}}\left(\vec{\alpha}_{E} \mathfrak{k}\right)\left(\vec{\alpha}_{n} \mathfrak{k}\right)
$$

durchführen. Schreibt man noch für:

$$
(\vec{\alpha} \mathfrak{l}) e^{ \pm i \mathfrak{k} x}= \pm i(\vec{\alpha} \nabla) e^{ \pm i \mathfrak{x}}
$$

so kann man an Stelle der Ströme mit Hilfe von (31) und (32), d.h. durch Benutzung der Kontinuitätsgleichungen und des $\mathrm{G}_{\mathrm{A}}$ ussschen Satzes, die Ladungen einführen. $U_{f i}(W)$ geht dann über in:

$$
\left.\begin{array}{l}
U_{f i}(W)=\sum_{n=1}^{A} e_{n} e_{E}\left\{\int \psi_{f}^{K^{*}} \psi_{f}^{E *} \frac{1}{\left|\mathrm{r}_{n}-\mathrm{r}_{E}\right|} \psi_{i}^{E} \psi_{i}^{K} d \tau_{K} d \tau_{E}+\right. \\
+\lim _{\sigma \rightarrow 0} \frac{1}{4 \pi^{2}}\left[\int \frac{d^{3} \mathfrak{f}}{k(W-k+i \sigma)} \int \psi_{f}^{E^{*}} \psi_{f}^{K^{*}}\left(\vec{\alpha}_{n} \vec{\alpha}_{E}-\frac{W^{2}}{k^{2}}\right) \times\right. \\
\times e^{i \mathfrak{f}\left(\mathfrak{x}_{E}-\mathfrak{r}_{n}\right)} \psi_{i}^{E} \psi_{i}^{K} d \tau_{E} d \tau_{K}- \\
\left.\left.-\int d^{3} \mathfrak{t} \frac{1}{k(W+k+i \sigma)} \int \psi_{f}^{E^{*}} \psi_{f}^{K *}\left(\vec{\alpha}_{n} \vec{\alpha}_{E}-\frac{W^{2}}{k^{2}}\right) e^{-i \mathfrak{k}\left(\mathfrak{r}_{E}-\mathfrak{r}_{n}\right)} \psi_{i}^{E} \psi_{i}^{K} d \tau_{E} d \tau_{K}\right]\right\} .
\end{array}\right\}
$$

Integration über die Richtung von $\mathfrak{f}$ und Zusammenfassen der beiden Integrale über in (62) ergibt:

$$
\left.\begin{array}{l}
U_{f i}(W)=\sum_{n=1}^{A} e_{n} e_{E}\left\{\int \psi_{f}^{K^{*}} \psi_{f}^{E^{*}} \frac{1}{\left|\mathfrak{r}_{n}-\mathfrak{r}_{E}\right|} \psi_{i}^{E} \psi_{i}^{K} d \tau_{E} d \tau_{K}+\right. \\
\left.+\lim _{\sigma \rightarrow 0} \frac{2}{\pi} \int_{0}^{\infty} \frac{k d k}{(W+i \sigma)^{2}-k^{2}} \int \psi_{f}^{K^{*}} \psi_{f}^{E^{*}}\left(\vec{\alpha}_{n} \vec{\alpha}_{E}-\frac{W^{2}}{k^{2}}\right) \frac{\sin k\left|\mathfrak{r}_{n}-\mathfrak{r}_{E}\right|}{\left|\mathfrak{r}_{n}-\mathfrak{r}_{E}\right|} \psi_{i}^{K} \psi_{i}^{E} d \tau_{K} d \tau_{E}\right\}
\end{array}\right\}
$$


Wie in $I$, wo $U_{f i}(W)$ durch Entwicklung von $\mathscr{A}(r)$ nach Multipolen berechnet wurde, tritt auch hier in dem Teil der Wechselwirkung, der durch den Austausch von virtuellen transversalen Photonen hervorgerufen wird, vor der Integration über die Energie dieser Photonen ein Term auf, der einen zusätzlichen Faktor $1 / k^{2}$ hat. Mit Hilfe von

$$
\frac{1}{k^{2}} \frac{1}{W^{2}-k^{2}}=\frac{1}{W^{2}}\left\{\frac{1}{W^{2}-k^{2}}+\frac{1}{k^{2}}\right\}
$$

können wir wieder diesen Term von dem anderen Resonanznenner $W^{2}-k^{2}$ abspalten. Damit läßt sich $U_{f i}(W)$ folgendermaßen schreiben:

$$
\begin{aligned}
& U_{f i}(W)=\sum_{n=1}^{A} e_{n} e_{E}\left\{\int \psi_{f}^{K^{*}} \psi_{f}^{E^{*}} \frac{1}{\left|\mathfrak{r}_{n}-\mathfrak{r}_{E}\right|} \psi_{i}^{K} \psi_{i}^{E} d \tau_{K} d \tau_{E}+\right. \\
& +\lim _{\sigma \rightarrow 0} \frac{2}{\pi}\left[\int_{0}^{\infty} \frac{k d k}{(W+i \sigma)^{2}-k^{2}} \int \psi_{f}^{K^{*}} \psi_{f}^{E^{*}}\left(\vec{\alpha}_{n} \vec{\alpha}_{E}-1\right) \frac{\sin k\left|\mathfrak{r}_{n}-\mathfrak{r}_{E}\right|}{\left|\mathfrak{r}_{n}-\mathfrak{r}_{E}\right|} \psi_{i}^{E} \psi_{i}^{K} d \tau_{K} d \tau_{E}-\right. \\
& \left.\left.-\int_{0}^{\infty} d k \int \psi_{f}^{E^{*}} \psi_{f}^{K^{*}} \frac{\sin k\left|\mathfrak{r}_{n}-\mathfrak{r}_{E}\right|}{k\left|\mathfrak{r}_{n}-\mathfrak{r}_{E}\right|} \psi_{i}^{E} \psi_{i}^{K} d \tau_{E} d \tau_{K}\right]\right\} .
\end{aligned}
$$

Nun ist:

$$
\int_{0}^{\infty} \frac{\sin k\left|\mathfrak{r}_{n}-\mathfrak{r}_{E}\right|}{k} d k=\frac{\pi}{2}
$$

Daher kompensiert das letzte Integral in (65) gerade das CovLomB-Matrixelement. Derselbe Sachverhalt ergab sich in I bei der Ableitung der Wechselwirkung mit Hilfe von Multipolen. Da der Pol bei $k=W$ in dem zweiten Glied in (65) in die obere Halbebene verschoben ist*, ergibt sich für dieses Integral über $k$ eine Exponentialfunktion mit positiv imaginärem Exponenten, nämlich":

$$
\int_{0}^{\infty} \frac{\sin k\left|\mathfrak{r}_{n}-\mathfrak{r}_{E}\right|}{(W+i \sigma)^{2}-k^{2}} k d k=-\frac{\pi}{2} e^{i W\left|\mathfrak{r}_{n}-\mathbf{r}_{E}\right|}
$$

Damit erhalten wir durch Einsetzen von (67) in (65) für $U_{f i}(W)$ die Gl. (56).

Das Übergangsmatrixelement für innere Konversion $U_{f i}(W)$ ist also bestimmt als Matrixelement des retardierten Wechselwirkungsoperators

$$
K=\sum_{n} e_{n} e_{E}\left(1-\vec{\alpha}_{n} \vec{\alpha}_{E}\right) \frac{e^{i W\left|\mathfrak{r}_{n}-\mathrm{r}_{E}\right|}}{\left|\mathrm{r}_{n}-\mathfrak{r}_{E}\right|}
$$

* Dies folgt aus der Störungsrechnung für gebundene Zustände, entwickelt in Heitlek, W.: Quantum Theory of Radiation, $\$ 16$ (1955).

4 Gröbner, W., u. N. Hofreiter: Integraltafel, zweiter Teil. 
Diesen Wechselwirkungsoperator können wir nach Kugelfunktionen entwickeln. Man erhältt ${ }^{5,6,7}$ :

$$
\begin{aligned}
& \left(1-\vec{\alpha}_{n} \vec{\alpha}_{E}\right) \frac{e^{i W\left|r_{n}-r_{E}\right|}}{\left|r_{n}-\mathfrak{r}_{E}\right|} \\
& \left.=\frac{2 \pi^{2} i}{W} \sum_{L, M, \tau}\left\{\psi_{L M}\left(W r_{E}\right) \Phi_{L M}^{*}\left(W r_{n}\right)-\vec{\alpha}_{E} \vec{B}_{L M}^{\tau}\left(W r_{E}\right) \vec{\alpha}_{n} \vec{A}_{L M}^{\tau^{*}}\left(W r_{n}\right)\right\}\right\}
\end{aligned}
$$

für $r_{n} \leqq \gamma_{E}$, und für $r_{n} \geq r_{E}$ den entsprechenden Ausdruck.

Die Summe über $\tau$ in (69) ist über $\tau=$ magnetisch, elektrisch und longitudinal zu erstrecken. Dabei ist (70) $\vec{A}_{L M}^{1}=\frac{1}{W} \nabla \Phi_{L M}$ bzw. $\vec{B}_{L M}^{\mathrm{l}}=\frac{1}{W} \nabla \psi_{L M}$. Die anderen Potentiale sind in (11), (24a) und (36) definiert. Setzen wir die Entwicklung (69) in (56) ein, unter Berücksichtigung der besonderen Gestalt der Entwicklung von $K$, je nachdem ob $r_{n} \leq r_{E}$ oder $r_{n} \geqq r_{E}$ ist, so gewinnen wir folgenden Ausdruck für $U_{i i}(W)$ :

$$
\begin{aligned}
& U_{f i}(W)=-\frac{2 \pi^{2} i}{W} \sum_{n=1}^{A} \sum_{L, M, \tau} e_{n} e_{E}\left\{\int_{0}^{R} \psi_{f}^{K *} \vec{\alpha}_{n} \vec{A}_{L M}^{\tau^{*}}\left(W \gamma_{n}\right) \psi_{i}^{K} d \tau_{K} \times\right. \\
& \times \int_{0}^{\infty} \psi_{f}^{E^{*}} \vec{\alpha}_{E} \vec{B}_{L M}^{\tau}\left(W \gamma_{E}\right) \psi_{i}^{E} d \tau_{E}+ \\
& +\int_{0}^{R}\left[\psi_{f}^{E^{*}} \vec{\alpha}_{E} \vec{A}_{L M}^{\tau}\left(W r_{E}\right) \psi_{i}^{E} \int_{\gamma_{E}}^{R} \psi_{f}^{K^{*}} \vec{\alpha}_{n} \vec{B}_{L M}^{z^{*}}\left(W r_{n}\right) \psi_{i}^{K} d \tau_{K}\right] d \tau_{E}- \\
& -\int_{0}^{R}\left\{\psi_{f}^{E^{*}} \vec{\alpha}_{E} \vec{B}_{L M}^{\tau}\left(W r_{E}\right) \psi_{i}^{E} \int_{\gamma_{E}}^{R} \psi_{f}^{K^{*}} \vec{\alpha}_{n} \vec{A}_{L M}^{\tau *}\left(W r_{n}\right) \psi_{i}^{K} d \tau_{K}\right] d \tau_{E}- \\
& -\int_{0}^{R} \psi_{f}^{K^{*}} \Phi_{L M}^{*}\left(W r_{n}\right) \psi_{i}^{K} d \tau_{K} \int_{0}^{\infty} \psi_{f}^{E^{*}} \psi_{L M}\left(W r_{E}\right) \psi_{i}^{E} d \tau_{E}- \\
& -\int_{0}^{R}\left[\psi_{f}^{E^{*}} \Phi_{L M}\left(W r_{E}\right) \psi_{i}^{E} \int_{\tau_{E}}^{R} \psi_{f}^{K *} \psi_{L M}^{*}\left(W r_{n}\right) \psi_{i}^{K} d \tau_{K}\right] d \tau_{E}+ \\
& \left.+\int_{0}^{R}\left[\psi_{f}^{E^{*}} \psi_{L M}\left(W \gamma_{E}\right) \psi_{i}^{E} \int_{\nu_{E}}^{R} \psi_{f}^{K^{*}} \Phi_{L M}^{*}\left(W r_{n}\right) \psi_{i}^{K} d \tau_{K}\right] d \tau_{E}\right\}
\end{aligned}
$$

Es besteht nun die Frage, $a b$ die in I entwickelten Gleichungen für

$$
U_{f i}(W)=U_{f i}^{(1)}(\text { magn })+U_{f i}^{(1)}(\text { elektr }),
$$

nämlich (24) und (38) mit (56) äquivalent sind. $U_{f i}^{(1)}$ (magn) stimmt bereits mit dem magnetischen Teil von (71) überein. Um $U_{f i}^{(1)}$ (elektr) d. h. I (38) aus (71) zu erhalten, müssen wir einige Umformungen machen.

5 Morse, P.M., u. H. Feshbach: Methods of Theoretical Physics, §9 (1946) bzw. Part. II, §13,3 (1953).

6 Tralli, N., u. G.H. Goertzel: Phys. Rev. 83, 399 (1951).

7 Rose, M. E.: Multipole Fields, §4,21 (1955). 
Dabei haben wir die Matrixelemente mit dem elektrischen, longitudinalen und skalaren Potential, die ja alle bei einem elektrischen Übergang $\psi_{i}^{K} \rightarrow \psi_{f}^{K}$ des Kern zu $U_{f i}(W)$ beitragen, zusammenzufassen.

Durch Anwendung der Kontinuitätsgleichung $\operatorname{div} \psi_{i}^{E^{*}} \vec{\alpha}_{E} \psi_{i}^{E}-$ $i W \psi_{f}^{E^{*}} \psi_{i}^{E}=0$ und partieller Integration formen wir das elektrische Potential in der Form (29) in die Form um, in der es in (38) erscheint. Beachtet man dabei, daß in den Integranden von (71) neben den Elektronenwellenfunktionen und den von $\mathfrak{r}_{E}$ abhängigen Potentialen noch eine jedoch nur von $\gamma_{E}$ abhängige Funktion steht, so geht der Teil von (71) der nur die elektrischen Potentiale enthält $\bar{U}_{f i}$ (elektr) über in:

$$
\bar{U}_{f i}(\text { elektr })=U_{f i}^{(1)}(\text { elektr })+\widetilde{U}_{f i}(\text { elektr })
$$

wo $U_{j i}^{(1)}$ (elektr) durch (38) gegeben ist und

$$
\begin{aligned}
& \widetilde{U}_{f i} \text { (elektr) }=\frac{2 \pi^{2} i}{W} \sum_{n=1}^{A} \sum_{L, M} e_{n} e_{E}\left\{\int_{0}^{R} \psi_{f}^{E *} \vec{\alpha}_{E} \mathfrak{r}_{E} r_{E} \psi_{i}^{E} \frac{d}{d \gamma_{E}} \gamma_{E} \frac{\psi_{L M}}{\sqrt{L(L+1)}} \times\right. \\
& \times\left[\int \psi_{f}^{K^{*}} \vec{\alpha}_{n} \vec{A}_{L M}^{\mathrm{e}^{*}}\left(W \gamma_{n}\right) \psi_{i}^{K} d \Omega_{K}\right]_{r_{n}=\gamma_{E}} d \tau_{E}- \\
& -\int_{0}^{R} \psi_{f}^{E^{*}} \vec{\alpha}_{E} \mathfrak{r}_{E} \gamma_{E} \psi_{i}^{E} \frac{d}{d \gamma_{E}} \gamma_{E} \frac{\Phi_{L M}}{\sqrt{L(L+1)}} \times \\
& \left.\times\left[\left.\int \psi_{f}^{K^{*}} \vec{\alpha}_{n} \vec{B}_{L M}^{\mathrm{e}^{*}}\left(W r_{n}\right) \psi_{i}^{K} d \Omega_{K}\right|_{r_{n}=\gamma_{E}} d \tau_{E}\right\}\right]
\end{aligned}
$$

$\left(d \Omega_{K}\right.$ ist das Oberflächenelement: $\left.d \tau_{1} d \tau_{2} \ldots d \tau_{n-1} d \Omega_{n} d \tau_{n+1} \ldots d \tau_{A}\right)$.

Setzt man in (73) die Definitionen für die Potentiale aus (11) und (38a) ein und führt die Differentiationen in den Potentialen aus, nämlich:

$$
\left.\begin{array}{rl}
\frac{d}{d r} r \Phi_{L M}= & \sqrt{\frac{2}{\pi}}\left(-L j_{L}+W r j_{L-1}\right) Y_{L M} \\
\vec{A}_{L M}^{\mathrm{el}}= & \frac{1}{\sqrt{\bar{L}(L+1)}} \sqrt{\frac{2}{\pi}} \times \\
& \times\left\{\left(-L j_{L}+W r j_{L-1}\right) \nabla Y_{L M}+L(L+1) \frac{j_{L}}{r} Y_{L M} \frac{\mathfrak{r}}{r}\right\},
\end{array}\right\}
$$

so erhält man für $\widetilde{U}_{f i}($ elektr):

$$
\begin{aligned}
& \widetilde{U}_{f i}(\text { elektr })=\frac{2 \pi^{2} i}{W} \sum_{n=1}^{A} \sum_{L, M} e_{n} e_{E} \frac{2}{\pi} W \times \\
& \times\left\{\int_{0}^{R} \psi_{f}^{E^{*}} \vec{\alpha}_{E} \psi_{i}^{E} \gamma_{E} \mathfrak{r}_{E} Y_{L M}\left(j_{L}\left(W r_{E}\right) h_{L-1}\left(W r_{E}\right)-h_{L}\left(W r_{E}\right) i_{L-1}\left(W r_{E}\right)\right) \times\right. \\
& \left.\times\left[\int \psi_{f}^{K^{*}} \vec{\alpha}_{n} \frac{\mathrm{r}_{n}}{v_{n}} Y_{L M}^{*} \psi_{i}^{K} d \Omega_{K}\right]_{r_{n}=r_{E}} d \tau_{E}\right\} .
\end{aligned}
$$


Wir betrachten jetzt:

$$
\begin{aligned}
U_{f i}(\text { long })= & -\frac{2 \pi^{2} i}{\bar{W}} \sum_{n=1}^{A} \sum_{L, M}\left\{\int_{0}^{R} \psi_{f}^{K^{*}} \vec{\alpha}_{n} \vec{A}_{L M}^{1^{*}} \psi_{i}^{K} d \tau_{K} \int_{0}^{\infty} \psi_{f}^{E^{*}} \vec{\alpha}_{E} \vec{B}_{L M}^{1} \psi_{i}^{E} d \tau_{E}+\right. \\
& +\int_{0}^{R}\left[\psi_{f}^{E^{*}} \vec{\alpha}_{E} \vec{A}_{L M}^{L} \psi_{i}^{E} \int_{\gamma_{E}}^{R} \psi_{f}^{K^{*}} \vec{\alpha}_{n} \vec{B}_{L M}^{1^{*}} \psi_{i}^{K} d \tau_{K}\right] d \tau_{E}- \\
& -\int_{0}^{R}\left[\psi_{f}^{E^{*}} \vec{\alpha}_{E} \vec{B}_{L M}^{1} \psi_{i}^{E} \int_{i}^{R} \psi_{f}^{K^{*}} \vec{\alpha}_{n} \vec{A}_{L M}^{1^{*}} \psi_{i}^{K} d \tau_{K}\right] d \tau_{E}- \\
& -\int_{0}^{R} \psi_{f}^{K^{*}} \Phi_{L M}^{*} \psi_{i}^{K} d \tau_{K} \int_{0}^{\infty} \psi_{f}^{E^{*}} \psi_{L_{M}} \psi_{i}^{E} d \tau_{E}- \\
& -\int_{0}^{R}\left[\psi_{f}^{E^{*}} \Phi_{L M} \psi_{i}^{E} \int_{\gamma_{E}}^{R} \psi_{f}^{K^{*}} \psi_{L M}^{*} \psi_{i}^{K} d \tau_{K}\right] d \tau_{E}+ \\
& \left.+\int_{0}^{R}\left[\psi_{f}^{E^{*}} \psi_{L M} \psi_{i}^{E} \int_{\gamma_{E}}^{R} \psi_{f}^{K^{*}} \Phi_{L M}^{*} \psi_{i}^{K} d \tau_{K}\right] d \tau_{E}\right\} .
\end{aligned}
$$

Auf Grund unserer Definitionen ist:

$$
\vec{A}_{L M}^{1}=\frac{1}{W} \nabla \Phi_{L M}=\sqrt{\frac{2}{\pi}}\left\{j_{L} \nabla Y_{L M}+\left[W j_{L-1}-(L+1) \frac{j_{L}}{r}\right] Y_{L M} \frac{\mathfrak{r}}{v}\right\}
$$

entsprechend für $\vec{B}_{L M}^{l}=\frac{1}{W} \nabla \psi_{L M}$.

Formt man $U_{f i}$ (long) in ähnlicher Weise wie $\bar{U}_{f i}$ (elektr) durch Anwendung der Kontinuitätsgleichungen:

$$
\operatorname{div} \psi_{f}^{E^{*}} \vec{\alpha}_{E} \psi_{i}^{E}-i W \psi_{f}^{E^{*}} \psi_{i}^{E}=0 \text { und } \operatorname{div} \psi_{f}^{K^{*}} \vec{\alpha}_{n} \psi_{i}^{K}+i W \psi_{f}^{K^{*}} \psi_{i}^{K}=0
$$

und partielle Integration um, so kompensieren sich die meisten Glieder in (76) und man erhält:

$$
\begin{aligned}
& U_{f i} \text { (long) }=-\frac{2 \pi^{2} i}{W} \sum_{n=1}^{A} \sum_{L, M} e_{n} e_{E} \times \\
& \quad \times\left\{\int_{0}^{R} \psi_{f}^{E *} \vec{\alpha}_{E} \psi_{i}^{E} \mathfrak{r}_{E} \frac{\gamma_{E}}{W} \Phi_{L M}\left[\int \psi_{f}^{K *} \vec{\alpha}_{n} \vec{B}_{L M}^{1^{*}} \psi_{i}^{K} d \Omega_{K}\right]_{r_{n}=r_{E}} d \tau_{E}-\right. \\
& \left.\quad-\int_{0}^{R} \psi_{j}^{E^{*}} \vec{\alpha}_{E} \psi_{i}^{E} \mathrm{r}_{E} \frac{\gamma_{E}}{W} \psi_{L M}\left[\int \psi_{f}^{K^{*}} \vec{\alpha}_{n} \vec{A}_{L M}^{1^{*}} \psi_{i}^{K} d \Omega_{K}\right]_{r_{n=\gamma_{E}}} d \tau_{E}\right\} .
\end{aligned}
$$

Diese Umformungen sind auch für den Grenzübergang $R \rightarrow 0$ für die Konversionskoeffizienten wichtig. Setzt man (78) für $\vec{A}_{L M}^{1}$ und den entsprechenden Ausdruck für $\vec{B}_{L M}^{1}$ in (79) ein, so wird $-U_{f i}$ (long) mit der rechten Seite von (75) identisch, wenn man von den Termen für $L=0$ absieht. Wir betrachten jetzt die Terme für $L=0$. 
Wegen $j_{L} k_{L-1}-h_{L} j_{L-1}=\frac{i}{W^{2} \gamma^{2}}$ vereinfacht sich $U_{f i}$ (long) nämlich noch zu:

$U_{f i}$ (long)
$\left.=\sum_{n=1}^{A} \sum_{L, M} e_{n} e_{E} \int_{0}^{R} \psi_{f}^{E *} \vec{\alpha}_{E} \psi_{i}^{E} \frac{\mathfrak{r}_{E}}{\gamma_{E}} Y_{L M}\left[\int \psi_{f}^{K^{*}} \vec{\alpha}_{n} \psi_{i}^{K} \frac{\mathfrak{r}_{n}}{\gamma_{n}} Y_{L M}^{*} d \Omega_{K}\right]_{r_{n}=\gamma_{E}} d \tau_{E} \cdot\right\}(79)$

Führen wir in

$$
\left.\begin{array}{r}
U_{i}^{(1)}(\mathrm{elektr})_{L=0}=\sum_{n=1}^{A} e_{n} e_{E}\left\{\int_{0}^{R}\left[\psi_{f}^{E^{*}} \psi_{i}^{E} \int_{\gamma_{E}}^{R} \psi_{f}^{K^{*}}-\frac{1}{\gamma_{n}} \psi_{i}^{K} d \tau_{K}\right] d \tau_{E}-\right. \\
\left.-\int_{0}^{R}\left[\psi_{f}^{E^{*}} \frac{1}{\gamma_{E}} \psi_{i}^{E} \int_{\nu_{E}}^{R} \psi_{f}^{K^{*}} \psi_{i}^{K} d \tau_{K}\right] d \tau_{E}\right\}
\end{array}\right\}
$$

wieder an Stelle der Ladungsverteilungen die Ströme ein, so erhalten wir nur noch ein einfaches Integral über die Elektronenkoordinaten nämlich:

$$
\begin{aligned}
& U_{f i}^{(1)}(\text { elektr) })_{L=0} \\
& =-\sum_{n=1}^{A} e_{n} e_{E}\left\{\frac { 1 } { W ^ { 2 } } \int _ { 0 } ^ { R } \psi _ { f } ^ { E * } \vec { \alpha } _ { E } \nabla ( \frac { 1 } { \gamma _ { E } } ) \psi _ { i } ^ { E } \gamma _ { E } ^ { 2 } \left[\left.\int \psi_{f}^{K *} \vec{\alpha}_{n} \frac{\mathfrak{r}_{n}}{\gamma_{n}} \psi_{i}^{K} d \Omega_{K}\right|_{\gamma_{n}=r_{\bar{H}}} d \tau_{E}\right.\right. \\
& =\sum_{n=1}^{A} e_{n} e_{E}\left\{\frac{1}{W^{2}} \int \psi_{f}^{E *} \vec{\alpha}_{E} \psi_{i}^{E} \frac{\mathfrak{r}_{E}}{\gamma_{E}}\left[\int \psi_{f}^{K *} \alpha_{n} \psi_{i}^{K} \frac{\mathfrak{x}_{n}}{\gamma_{n}} d \Omega_{K}\right]_{r_{n}=\gamma_{H}} d \tau_{E}\right\}
\end{aligned}
$$

Wegen $Y_{00}^{2}=\frac{1}{4 \pi}$ ist aber (81) mit den Termen für $L=0$ von (80) identisch. Damit haben wir insgesamt gezeigt, daß sich $U_{i i}(W)$ nach (56) in die in I hergeleiteten Gleichungen für $U_{i i}^{(1)}$ (magn) und $U_{f i}^{(1)}$ (elektr) umformen läßt.

Durch die Umformungen erscheint der Grenzübergang zu einem punktförmigen Kern für die Konversionskoeffizienten noch in einem anderen Licht. Macht man nämlich in dem Konversionskoeffizienten $\left.N_{e}\left|N_{q}=\right| U_{f i}(W)\right|^{2} / N_{q}$ wo $N_{q}$ durch (14) gegeben ist und $U_{f i}(W)$ in der Gestalt (71), rein formal den Grenzübergang $R \rightarrow 0$, so treten in diesem Ausdruck divergente Integrale auf, und zwar bei den Matrixelementen, die das elektrische und das longitudinale Potential enthalten. Fassen wir aber diese beiden Arten von Matrixelementen durch die ausgeführten Umformungen zusammen, so ergibt der Grenzübergang das endliche Resultat (47) in I. 